5. Grandas F, Quinn N, Critchley P, Rohan A, Marsden CD, Stahl SM. Antiparkinsonian activity of a single oral dose of PHNO. Mov Disord. 1987;2: 47-51.

6. Coleman RJ, Quinn NP, Traub M, Marsden CD. Nasogastric and intravenous infusions of (+)-4-propyl-9-hydroxynaphthoxazine (PHNO) in Parkinson's disease. J Neurol Neurosurg Psychiatry. 1990;53:102-105.

7. Cedarbaum JM, Clark M, Toy LH, Green-Parsons A. Sustained-release (+)-PHNO [MK-458 (HPMC)] in the treatment of Parkinson's disease: evidence for tolerance to a selective $\mathrm{D}_{2}$-receptor agonist administered as a long-acting formulation. Mov Disord. 1990;5:298-303.

\author{
Romina Mizrahi* \\ Sylvain Houle \\ Irina Vitcu \\ Alvina Ng \\ Alan A Wilson \\ *PET Centre, CAMH \\ University of Toronto \\ 250 College St. \\ Toronto, ON, M5T 1R8, Canada \\ E-mail: romina.mizrahi@camhpet.ca
}

DOI: $10.2967 /$ jnumed.109.072314

\section{The Twilight Saga of Insulin Administration in Hyperglycemic Patients Undergoing ${ }^{18}$ F-FDG PET}

TO THE EDITOR: Roy et al. recently published a study adopting a standardized protocol of intravenous insulin administration to reduce glycemia in diabetic cancer patients undergoing ${ }^{18} \mathrm{~F}$-FDG PET (1). The authors claimed that the pretest intravenous insulin injection in diabetic patients is a realistic approach. However, several issues deserve further exploration before this standardized insulin protocol can be incorporated into daily PET practice.

In clinical tumor imaging, hyperglycemia has a recognized adverse effect on the quality of ${ }^{18}$ F-FDG PET images because of competitive inhibition of ${ }^{18} \mathrm{~F}-\mathrm{FDG}$ uptake by glucose. Although insulin can be used as a glycemia-reducing agent, arbitrary prescription of insulin before ${ }^{18} \mathrm{~F}$-FDG injection may exacerbate muscular ${ }^{18} \mathrm{~F}$-FDG uptake and compromise tumor uptake, thus curtailing image interpretability (2). According to the study results of Roy et al., ${ }^{18}$ F-FDG PET image quality was barely adequate in $75 \%$ of patients receiving insulin. This means every 1 of 4 scans must be repeated. Repeating a study is not a cost-benefit if the PET center does not have its own on-site cyclotron. Rescheduling is inconvenient to the patients and bothersome to the center staff. The set point to prescribe insulin in the study protocol of Roy et al. might account for their poor image quality. The Society of Nuclear Medicine recommends rescheduling the examination if the patient's blood glucose level is greater than $8.3-11.1 \mathrm{mmol} / \mathrm{L}$ (150-200 mg/dL) (3). The European Association of Nuclear Medicine also advises that an ${ }^{18}$ F-FDG PET study should not be performed when the blood glucose level exceeds $11.1 \mathrm{mmol} / \mathrm{L}$ (4). If cancelling an examination or rescheduling an appointment is not feasible, we suggest the use of intravenous insulin at a blood glucose level of more than $11.1 \mathrm{mmol} / \mathrm{L}$, instead of the $10.0 \mathrm{mmol} / \mathrm{L}$ stated by Roy et al. Additionally, we encourage hyperglycemic patients to have a temperate walk after insulin injection to reduce muscular uptake. In this way, the proportion of images of adequate quality would improve.
In their study, less favorable image quality was found with more glycemic reduction after insulin administration, and no significant correlation was observed between muscular uptake and parameters such as initial glycemia, total insulin dose, and number of insulin doses. Hence, the extent of glycemic reduction is not predictable and the chance of study failure is unavoidable. The implication is that we cannot select which hyperglycemic patient is suitable for insulin use. We also cannot apply the correct insulin dose to manage glycemic reduction before imaging. These phenomena can be explained by nonuniform insulin sensitivity among hyperglycemic patients. Therefore, Roy's standardized insulin protocol, an illogical practice such as sliding-scale insulin (5), is a problematic recipe for glycemic control in hyperglycemic patients undergoing ${ }^{18} \mathrm{~F}$-FDG PET studies. Sliding-scale insulin is also associated with poorer glycemic control, a harmful rollercoaster effect between hyperglycemic and hypoglycemic episodes, and increased risks of hypoglycemia, as occurred in 6 patients $(9.5 \%)$ in the study of Roy et al. Thus, their standardized insulin protocol might be a risky strategy.

To obtain a useful diagnostic image of ${ }^{18} \mathrm{~F}$-FDG PET, one should ensure that the patient has fasted overnight and has a blood glucose level of less than $8.3 \mathrm{mmol} / \mathrm{L}$ in the early morning on the day of the PET scan. A good method is to do a "practice run" by checking the patient's blood glucose levels for at least $3 \mathrm{~d}$ before the ${ }^{18} \mathrm{~F}-\mathrm{FDG}$ PET appointment (6). If the morning blood glucose level is persistently higher than $8.3 \mathrm{mmol} / \mathrm{L}$, the scheduler needs to recognize this problem well before the scan appointment and request that the diabetologist manage the glycemic status by basal and nutritional insulin therapy with a supplemental insulin regimen (7). To avert the possibility of irreversibly unreadable images, hypoglycemia, and transcellularshift hypokalemia, before establishing specific guidelines for using insulin in hyperglycemic patients undergoing ${ }^{18} \mathrm{~F}-\mathrm{FDG}$ PET we should have the patients fast and we should not administer additional insulin.

\section{REFERENCES}

1. Roy F-N, Beaulieu S, Boucher L, Bourdeau I, Cohade C. Impact of intravenous insulin on ${ }^{18}$ F-FDG PET in diabetic cancer patients. J Nucl Med. 2009;50:178183

2. Coleman RE. Clinical PET in oncology. Clin Positron Imaging. 1998;1:15-30.

3. Delbeke D, Coleman RE, Guiberteau MJ, et al. Procedure guideline for tumor imaging with ${ }^{18} \mathrm{~F}$-FDG PET/CT 1.0. J Nucl Med. 2006;47:885-895.

4. Bombardieri E, Aktolun C, Baum RP, et al. FDG-PET: procedure guidelines for tumour imaging. Eur J Nucl Med Mol Imaging. 2003;30:BP115-BP124.

5. Umpierrez GE, Palacio A, Smiley D. Sliding scale insulin use: myth or insanity? Am J Med. 2007;120:563-567.

6. Hamblen SM, Lowe VJ. Clinical ${ }^{18}$ F-FDG oncology patient preparation techniques. J Nucl Med Technol. 2003;31:3-7.

7. Clement S. Better glycemic control in the hospital: beneficial and feasible. Cleve Clin J Med. 2007;74:111-112, 114-120.

\author{
Yuh-Feng Lin \\ Fu-Chiu Yu \\ Jainn-Shiun Chiu* \\ *Taipei Medical University-Shuang Ho Hospital \\ No. 291, Zhongzheng Rd. \\ Zhonghe City, Taipei County 235, Taiwan \\ E-mail:shiunkle@mail2000.com.tw
}

DOI: 10.2967/jnumed.109.070185 